\title{
The fate of the bone graft in cerebrospinal fluid rhinorrhea endoscopic repair for idiopathic intracranial hypertension: a retrospective case series analysis*
}

\author{
Alan D. Workman', Ryan M. Carey'1, Arjun K. Parasher', Edward C. Kuan'1, $\quad$ Rhinology 55: 376-381, 2017 \\ Joe Godovchik², Jordan T. Glicksman', David W. Kennedy', James N. Palmer', https://doi.org/10.4193//hhino17.147 \\ Nithin D. Adappa'
}

' Department of Otorhinolaryngology: Head and Neck Surgery, University of Pennsylvania, Perelman School of Medicine,

Philadelphia, PA, USA

*Received for publication:

July 7, 2017

Accepted: October 5, 2017

${ }^{2}$ Sidney Kimmel Medical College at Thomas Jefferson University, Philadelphia, PA, USA

\begin{abstract}
Introduction: Idiopathic intracranial hypertension (IIH) is a common cause of spontaneous cerebrospinal fluid (CSF) leaks necessitating surgical intervention, and grafting of septal, mastoid, or turbinate bone over the defect is increasingly performed to strengthen the repair of the primary defect. However, the postoperative fate of these grafted bone fragments is largely unknown.

Methodology: We performed a retrospective study of patients at the University of Pennsylvania undergoing repair of spontaneous CSF leaks secondary to IIH. Preoperative and postoperative CT's were analyzed to determine the integration status of the transplanted bone.
\end{abstract}

Results: Fourteen patients with IIH and spontaneous CSF leak were analyzed, with a mean postoperative imaging follow-up period of four years. Thirteen patients (93\%) had bone present on CT imaging, with 11 of these patients displaying evidence of bone integration. Two patients (14\%) had a recurrent CSF leak in the same area, including the patient with absence of bone on imaging follow-up.

Conclusions: Bone grafts frequently incorporate when used for repair of spontaneous CSF leaks associated with IIH. The rate of incorporation is comparable to bone grafts used for other etiologies of CSF leak, despite the increased pressure on the repair site. Any rigid repair of the leak site should likely be accompanied by treatment of the underlying intracranial hypertension to avoid leak recurrence.

Key words: Idiopathic intracranial hypertension, bone graft, CSF leak, CSF leak repair, skull base surgery

\section{Introduction}

Idiopathic intracranial hypertension (IIH), also known as pseudotumor cerebri or benign intracranial hypertension, is a syndrome of unknown etiology diagnosed by an elevated intracranial pressure (ICP) in an alert patient with papilledema, in the presence of normal neuroimaging and cerebrospinal fluid (CSF) composition ${ }^{(1)}$. Patients have no focal neurologic deficits (except an abducens nerve palsy in some individuals) and may experience visual disturbances, headaches, and tinnitus. It is not uncommon for patients to be entirely asymptomatic ${ }^{(2)}$. IIH is ultimately distinguished from other ICP-related pathologies via exclusion according to the Dandy criteria ${ }^{(1,3)}$. The incidence of IIH is approximately 1 in 100,000 persons ${ }^{(1)}$, increasing to 3.5 of 100,000 in women aged 15-44 years ${ }^{(4)}$, and 22 of 100,000 in women aged $15-44$ years who are obese ${ }^{(5)}$. Correspondingly, the overwhelming majority of IIH patients (90\%) are obese female individuals of childbearing age ${ }^{(6)}$. Due to pressure-induced optic nerve damage, permanent vision loss or impairment are significant threats for these patients, occurring in $31 \%$ of $\operatorname{cases}^{(7-13)}$. IIH is becoming an increasingly diagnosed cause of spontaneous 
CSF leaks, particularly in CSF rhinorrhea ${ }^{(4,14)}$. This, paired with the association between $\mathrm{IIH}$ and obesity, suggests that the prevalence of IIH may increase as obesity becomes an even more ubiquitous disease $\mathrm{e}^{(6,15)}$.

Due to the severity and irreversible nature of some IIH-related complications, surgical repair is often performed. Endoscopic endonasal repairs of skull base defects due to IIH have failure rates ranging between 25 and $40 \%$, compared to a failure rate of $<10 \%$ for defects resulting from other etiologies ${ }^{(16-20)}$. These failures often recur at the site of repair or at another site along the skull base ${ }^{(17,21,22)}$. One surgical technique assumed to strengthen these repairs and possibly help prevent recurrence of the defect is the grafting of septal, mastoid, or middle turbinate bone within a multilayered repair technique ${ }^{(23)}$. However, analysis has shown that the implementation of these bone grafts in repairing IIH-related defects was not significantly associated with nonrecurrence of the defect ${ }^{(24)}$. Of course, this observation could be due to the selective implementation of the bone grafts in more severe cases. Therefore, analysis of the postoperative fate of these grafted bone fragments may help in determining if they actually contribute to the integrity and stability of the repair in the long term. Our goal was to observe if the bone graft was still present at the repair site postoperatively, and if so, whether the bone was free-floating or had become incorporated into the native bone tissue. We conducted a retrospective review of CSF repair patients who had pre and postoperative CT scans available and reported on the presence and condition of the transplanted bone.

\section{Materials and methods}

This study was a retrospective chart review of patients seen by the Division of Rhinology at the University of Pennsylvania between 2003 and 2015. Patients were included in the study if they were determined to have 1) CSF rhinorrhea confirmed by a positive $\beta-2$ transferrin test, 2 ) imaging evidence of a skull base defect, and 3) a diagnosis of IIH by the modified Dandy criteria ${ }^{(3)}$ or if ICP measurements were not available, a diagnosis of intracranial hypertension of unknown cause. Furthermore, patients included in the study were required to have preoperative CT imaging, surgical repair using a bone graft, and post-operative imaging available at least 1 year following surgery. We did not perform a post surgical CT scan for evaluation of the bone graft, but rather for other indications, including chronic rhinosinusitis work-up or concerns for additional recurrence. The study was approved by the Institutional Review Board at the University of Pennsylvania.

All CSF leaks were repaired by a fellowship-trained rhinologist using an endoscopic approach. Lumbar drains were placed prior to surgery. After obtaining opening pressures, intrathecal fluo- rescein was used to localize the skull base defect(s). Endoscopic dissection was performed to expose the defect and bipolar cautery was used to reduce encephaloceles. A multilayered technique was used for repair including a combination of fascial underlay, bone, and a vascularized mucosal flap or free mucosal graft. All grafts were underlay grafts, generally the same size or slightly smaller than the bony defect itself. Further, the mucosal overlay was sized to cover 360 degrees of the exposed skull base in order to achieve adherence. The bone graft was harvested from a number of different locations including the nasal septum, turbinates, or mastoid. Additional materials used in the repairs included Gelfoam (Pfizer, New York, NY, USA), Tisseel (Baxter Inter- national Inc., Deerfield, IL, USA), and Merocel packing (Medtronic, Jacksonville, FL, USA).

Patient demographic and disease characteristics were collected for analysis. Pre-operative and post-operative CTs were compared to determine the fate of the bone graft used to repair the skull base defect. The bone graft was first determined to be "present" or "absent" at the repair site in the follow up imaging. If present, the bone was classified as either "integrated," meaning it had been incorporated into the native bone tissue, or "not integrated" if the bone was free-floating. Radiographs were critically evaluated for fracture lines or complete callus formation (union) between grafted bone and native skull base utilizing established methodology used to evaluate quality of mandibular bony union following reconstruction ${ }^{(25)}$.

Statistical analyses were performed using STATA/IC 13.0 software (Stata Corporation, College Station, TX, USA).

\section{Results}

Between 2003 and 2015, ninety-nine patients underwent surgical repair of CSF leaks for IIH. Of those patients, 14 met inclusion criteria, most importantly having adequate imaging available for analysis (Table 1). The breakdown of excluded patients showed 77 individuals who did not have adequate imaging, and 8 patients with defects repaired without mention of bone grafting in the operative report. Of the 14 patients meeting criteria for the study, $85.7 \%$ were female and the mean age at the time of first surgery was $47.8 \pm 12.8$ years. Mean body mass index (BMI) at first surgery was $35.6 \pm 6.6 \mathrm{~kg} / \mathrm{m}^{2}$. All but one patient received a lumbar drain pre-operatively. The mean imaging follow-up for bone graft analysis after surgery was $3.97 \pm 3.58$ years, and mean clinical follow up was $4.68 \pm 3.83$ years. All patients had encephaloceles at presentation. The majority of patients (92.9\%) were maintained on acetazolamide postoperatively.

Thirteen of the patients had a single skull base defect; one presented with two simultaneous, separate leaks of the ethmoid roof and lateral recess of the sphenoid. The locations of skull 
Table 1. Patient demographics and clinical data.

\begin{tabular}{|lc|}
\hline & Patients $(\mathbf{n}=\mathbf{1 4})$ \\
\hline Age, mean (SD), y & $47.8 \pm 12.8$ \\
\hline Female, \% & 85.7 \\
\hline BMl, mean (SD) kg/m² & $35.6 \pm 6.6$ \\
\hline Ethnicity, \% & 64.3 \\
\hline White & 7.1 \\
\hline Black & 28.6 \\
\hline Other & 21.4 \\
\hline Prior sinus surgery, \% & 14.3 \\
\hline History of meningitis, \% & 92.9 \\
\hline Acetazolamide, \% & 21.4 \\
\hline VPS, \% & $2(14.3)$ \\
\hline Recurrence, no. (\%) & \\
\hline
\end{tabular}

Table 2. Skull base defect location.

\begin{tabular}{|l|l|}
\hline Cribriform & No. (\%) \\
\hline Frontal sinus & $(21.4)$ \\
\hline Frontal recess & $2(14.2)$ \\
\hline Ethmoid roof & $3(21.4)$ \\
\hline Sphenoid & $2(14.3)$ \\
\hline Central & $1(7.1)$ \\
\hline Posterior & $3(21.4)$ \\
\hline Lateral recess & $1(7.1)$ \\
\hline Sphenoid and ethmoid &
\end{tabular}

Table 3. Bone graft location and fate.

\section{No. (\%)}

\begin{tabular}{|lc|}
\hline Bone graft location & No. $(\%)$ \\
\hline Nasal septum & $7(50.0)$ \\
\hline Middle turbinate & $5(35.7)$ \\
\hline Inferior turbinate & $1(7.1)$ \\
\hline Mastoid tip & $1(7.1)$ \\
\hline Bone fate & $13(92.9)$ \\
\hline Bone present & $11(78.6)$ \\
\hline Bone integrated
\end{tabular}

base defects are listed in Table 2, and bone graft sources are listed in Table 3. Intraoperative size of skull base defect was listed in 10 cases, ranging from $0.16 \mathrm{~cm}^{2}$ to $3.78 \mathrm{~cm}^{2}$ with an average defect size of $1.45 \mathrm{~cm} 2 \pm 1.43 \mathrm{~cm}^{2}$. Two patients (14.3\%) had recurrent CSF leaks necessitating additional surgery. Three patients, including the two with recurrences, received ventriculoperitoneal shunts (VPS). Both re-leaks were in the same locations as the initial defects.

All but one patient had bone present on post-operative imaging. Eleven of the thirteen patients with bone present also had evidence of bone integration into the skull base at the location of the defect (Figure 1). Two patients had bone graft present, without integration into the skull base (Figure 2). Both non-integrated bone grafts were harvested from the nasal septum and used for defects of the cribriform and ethmoid roof, respectively.

The patient with absence of bone at the site of bone graft placement had a CSF leak recurrence within a year of the initial surgery. This patient's first repair of the left cribriform plate included a nasal septal flap with bone graft from the septum and was performed without fluorescein as a lumbar drain was unable to be placed preoperatively. After the re-leak, a VPS was placed and eventually a second surgery with endoscopic and bicoronal approach was performed. Over a year after the second surgery, the patient had not had any additional recurrences.

The other patient with a recurrence had evidence of bone integration on follow up imaging. This patient's first surgery included a repair of the posterior sphenoid using nasal septum and free mucosa. Within a month of the initial surgery, the patient had a recurrent leak and underwent VP placement. The patient then underwent endoscopic repair with temporalis fascia and muscle graft. The patient was without recurrence for greater than 10 years after the second repair.

\section{Discussion}

IIH is a frequent cause of spontaneous CSF leaks ${ }^{(4,14)}$, and failure rates of repair are higher in $\mathrm{IH}$-triggered leaks than those originating from other etiologies ${ }^{(20,26)}$. Bone grafting is increasingly used to strengthen the site of repair, as defects tend to recur at the primary site or an adjacent area of the skull base, and spontaneous osseous closure of defects within the skull base tends not to occur naturally ${ }^{(17,21,22,27,28)}$. However, the fate of the grafted bone is relatively unknown in cases of $\mathrm{IIH}$; the graft itself may integrate into native bone, remain free-floating, or disappear entirely. To describe the fate of this bone, we report on a representative cohort of 14 patients with imaging followup at an average of 4 years following primary surgery. Overall, the majority of patients (79\%) had bone integration, with an additional 14\% displaying free-floating bone. One out of the 14 

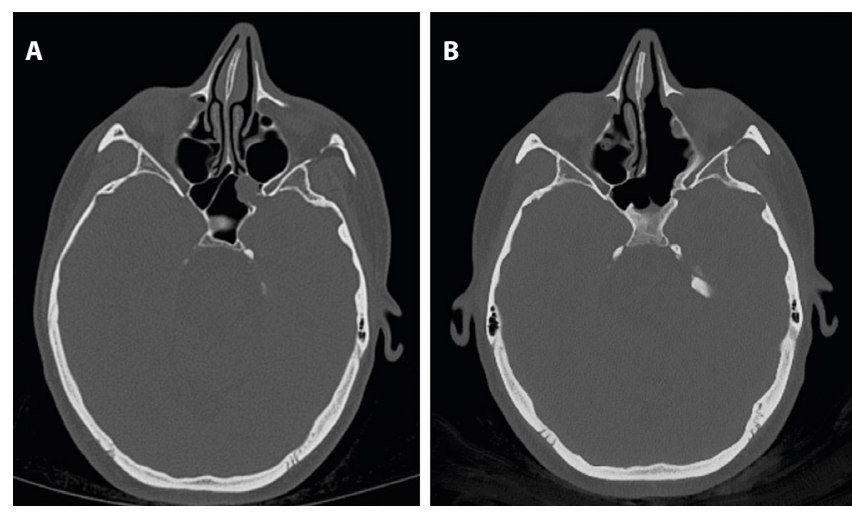

Figure 1. A) Preoperative computed tomography (CT) of a patient with idiopathic intracranial hypertension with a left sphenoid lateral recess skull base defect and meningoencephalocele. B) Postoperative CT demonstrating bone graft that is present and integrated.

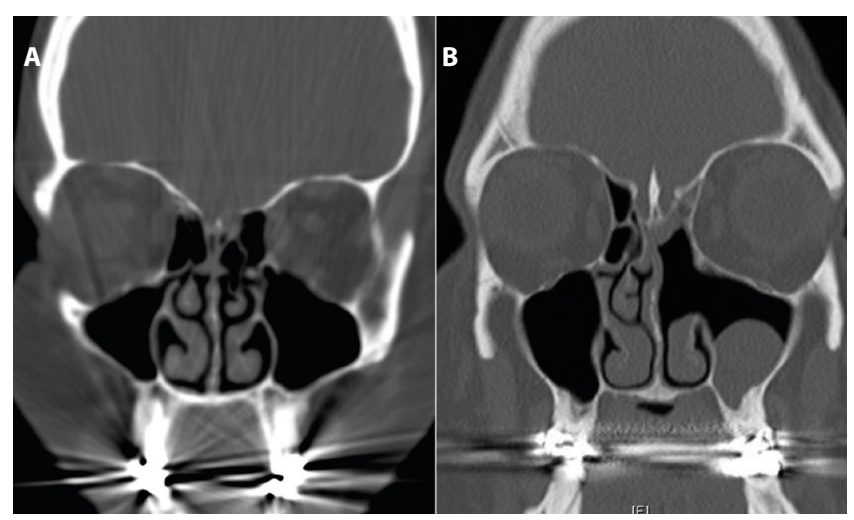

Figure 2. A) Preoperative computed tomography (CT) of a patient with idiopathic intracranial hypertension with a left cribriform plate skull base defect and meningoencephalocele. B) Postoperative CT demonstrating bone graft that is present but not integrated. patients had no evidence of bone at the time of imaging followup, and this patient was one of two patients who experienced a recurrent CSF leak during the follow-up period.

The demographic characteristics of the patients included in our study were consistent with those observed in IIH generally; a large majority of individuals were female and the mean BMI was in the morbidly obese range. The most common locations of spontaneous CSF leaks are the lateral recess of the sphenoid, the cribriform plate, and the roof of the ethmoid, which are also those observed in our cohort ${ }^{(19,29,30)}$. Our rate of recurrence was comparable to that observed by Campbell et al. in a prior investigation, a study that also demonstrated that same-site recurrences tend to recur earlier than those occurring in a separate location ${ }^{(24)}$. Indeed, the two recurrences in our study both occurred in the first year after primary surgery, and in the same location as the initial defect.

It is important to report the results of skull base bone grafts in $\mathrm{IIH}$ in the context of those performed for other causes, as the high-pressure environment of $\mathrm{IH}$ may account for the increased rate of re-leakage ${ }^{(20)}$. Bone grafts theoretically help to restore skull base continuity and rigidly resist this pressure while healing occurs ${ }^{(28)}$, but this increased pressure may also prevent integration of the bone that may be observed in other cases. A study of bone graft repair in all-cause spontaneous CSF leak by Ramakrishnan et al. demonstrated $62 \%$ bone incorporation, $23 \%$ incomplete incorporation, and $15 \%$ bone absence on postoperative imaging evaluation at a mean of 3.7 years ${ }^{(28)}$. As a similar or greater rate of bone incorporation was observed in our cohort of IIH patients, it can be hypothesized that the high-pressure environment of IIH does not prevent proper bone assimilation.
Ideal treatment of spontaneous CSF leaks in IIH consists of repair appropriate to the defect size ${ }^{(31)}$ in addition to aggressive management of the underlying intracranial hypertension. With a rigid endoscopic reconstruction and successful treatment of elevated ICP, success rates of repair have been shown to approach those observed in other etiologies of CSF leak. We note that while the bone graft provides additional strength to an area of weakness in the skull base, it does not necessarily improve watertight closure. In our cohort, almost all patients were maintained postoperatively on acetazolamide, with an additional subset of patients receiving VPS. Increased intracranial pressure has been associated with non-closure of leak repair sites $^{(32)}$, and patients with episodic rises in ICP, such as during apneic events ${ }^{(33)}$, may be even more appropriate for aggressive management ${ }^{(34)}$. Accumulation of truncal adiposity is thought to elevate central venous pressure, cerebral venous pressure, and ICP(35-37); therefore, weight loss is crucial for all obese IIH patients, as even modest weight fluctuations can significantly impact many clinical symptoms ${ }^{(38-40)}$. In fact, Campbell et al. showed that the BMI of patients in almost all cases of CSF leak recurrence was the same or had increased by the time of the second surgery ${ }^{(24)}$. Additional investigations may help provide some clarity on the role of lifestyle management to prevent recurrences in this specific population.

Some limitations of our study are the relatively small sample size, inconclusive evidence that bone graft absence is definitively associated with long-term recurrence, and difficulty of radiographic evaluation for bony union in small bone grafting. Additionally, the patient population receiving bone grafts in a retrospective study may inherently have more serious initial defects, perhaps explaining previous findings showing that bone grafting does not conclusively prevent re-leak ${ }^{(24)}$. Individuals with IIH who undergo repair of CSF leak without bone grafting 
could not be used as a potential control group in this study, due to inherent differences in surgical factors including defect size and severity of leak. Finally, bone graft placement should only be performed in cases where the surrounding bone is stable enough for support of the graft, without increasing risk of local fracture.

\section{Conclusion}

Overall, we demonstrate that bone grafts frequently incorporate when used in repair for treatment of spontaneous CSF leaks associated with IIH. CSF leak recurrence may be associated with absence of bone graft on follow-up imaging, but further investigations are needed to support this claim. Knowledge of bone integration status may be valuable to clinicians interested in advising their patients on resumption of strenuous activity, including resistance training or scuba diving. In IIH patients, it is critical to address both the need for a rigid repair of the defect as well as a reduction in intracranial pressures to adequately prevent leak recurrence.

\section{Authorship contribution}

ADW, RMC, and JG wrote the manuscript, ADW, RMC, AKP, ECK, JTG, DWK, JNP, NDA performed data collection and analyzed data, DWK, JNP, NDA, and AKP are responsible for the conceptualization and substantial contribution to design of the study. All authors critically revised the manuscript and gave their final approval.

\section{Conflict of interest}

None.

\section{References}

1. Thurtell MJ, Bruce BB, Newman NJ, et al. An update on idiopathic intracranial hypertension. Rev Neurol Dis 2010;7:e56-68.

2. Galvin JA, Van Stavern GP. Clinical characterization of idiopathic intracranial hypertension at the Detroit Medical Center. J Neurol Sci 2004;223:157-60.

3. Friedman DI, Jacobson DM. Diagnostic criteria for idiopathic intracranial hypertension. Neurology 2002;59:1492-5

4. Wise SK, Schlosser RJ. Evaluation of spontaneous nasal cerebrospinal fluid leaks. Curr Opin Otolaryngol Head Neck Surg 2007;15:28-34.

5. Kilgore KP, Lee MS, Leavitt JA, et al Re-evaluating the Incidence of Idiopathic Intracranial Hypertension in an Era of Increasing Obesity. Ophthalmology 2017:124:697-700.

6. Wall M. Idiopathic intracranial hypertension. Neurol Clin 2010;28:593-617.

7. Rowe FJ, Sarkies NJ. Assessment of visual function in idiopathic intracranial hypertension: a prospective study. Eye (Lond) 1998;12 ( Pt 1):111-8.

8. Wall M, George D. Visual loss in pseudotumor cerebri. Incidence and defects related to visual field strategy. Arch Neurol 1987;44:170-5.

9. Orcutt JC, Page NG, Sanders MD. Factors affecting visual loss in benign intracranial hypertension. Ophthalmology 1984;91:1303-12.

10. Corbett JJ, Savino PJ, Thompson HS, et al Visual loss in pseudotumor cerebri. Followup of 57 patients from five to 41 years and a profile of 14 patients with permanent severe visual loss. Arch Neurol 1982;39:46174

11. Bruce BB, Preechawat P, Newman NJ, et al Racial differences in idiopathic intracranial hypertension. Neurology 2008;70:861-7.

12. Radhakrishnan K, Ahlskog JE, Cross SA, et al. Idiopathic intracranial hypertension (pseudotumor cerebri). Descriptive epidemiol- ogy in Rochester, Minn, 1976 to 1990. Arch Neurol 1993;50:78-80.

13. Rowe FJ, Sarkies NJ. Visual outcome in a prospective study of idiopathic intracranial hypertension. Arch Ophthalmol 1999:117:1571.

14. Curry WT, Jr., Butler WE, Barker FG, 2nd. Rapidly rising incidence of cerebrospinal fluid shunting procedures for idiopathic intracranial hypertension in the United States, 1988-2002. Neurosurgery 2005:57:97-108; discussion 97-08.

15. Ogden CL, Carroll MD, Kit BK, et al. Prevalence of childhood and adult obesity in the United States, 2011-2012. JAMA 2014;311:806-14.

16. Schick $B$, Ibing $R$, Brors $D$, et al. Long-term study of endonasal duraplasty and review of the literature. Ann Otol Rhinol Laryngol 2001;110:142-7.

17. Gassner HG, Ponikau JU, Sherris DA, et al CSF rhinorrhea: 95 consecutive surgical cases with long term follow-up at the Mayo Clinic. Am J Rhinol 1999;13:439-47.

18. Hubbard JL, McDonald TJ, Pearson BW, et al. Spontaneous cerebrospinal fluid rhinorrhea: evolving concepts in diagnosis and surgical management based on the Mayo Clinic experience from 1970 through 1981. Neurosurgery 1985;16:314-21.

19. Schlosser RJ, Wilensky EM, Grady MS, et al. Elevated intracranial pressures in spontaneous cerebrospinal fluid leaks. Am J Rhinol 2003;17:191-5.

20. Lindstrom DR, Toohill RJ, Loehrl TA, et al. Management of cerebrospinal fluid rhinorrhea: the Medical College of Wisconsin experience. Laryngoscope 2004;114:969-74.

21. Carrau RL, Snyderman CH, Kassam AB. The management of cerebrospinal fluid leaks in patients at risk for high-pressure hydrocephalus. Laryngoscope 2005;115:205-12.

22. Wang EW, Vandergrift WA, 3rd, Schlosser RJ. Spontaneous CSF Leaks. Otolaryngol Clin North Am 2011:44:845-56, vii.

23. Schlosser RJ, Bolger WE. Nasal cerebrospinal fluid leaks: critical review and surgical considerations. Laryngoscope 2004;114:255-65.

24. Campbell RG, Farquhar D, Zhao N, et al Cerebrospinal fluid rhinorrhea secondary to idiopathic intracranial hypertension: Longterm outcomes of endoscopic repairs. Am J Rhinol Allergy 2016;30:294-300.

25. Akashi M, Hashikawa K, Kakei Y, et al. Sequential evaluation for bone union of transferred fibula flaps in reconstructed mandibles: panoramic X-ray versus computed tomography. Int J Oral Maxillofac Surg 2015;44:942-7.

26. Schlosser RJ, Bolger WE. Spontaneous nasal cerebrospinal fluid leaks and empty sella syndrome: a clinical association. Am J Rhinol 2003;17:91-6.

27. Brain C Lobo MMB, Rick F Nelson. Surgical Repair of Spontaneous Cerebrospinal Fluid (CSF) Leaks: A Systematic Review. Laryngoscope Investigative Otolaryngology 2017.

28. Ramakrishnan VR, Terella AM, Poonia S, et al. Osseous Repair in Minimally Invasive Reconstruction of Anterior Skull Base Defects. J Craniofac Surg 2017;28:36-39.

29. Seth R, Rajasekaran K, 3rd, Luong A, et al. Spontaneous CSF leaks: factors predictive of additional interventions. Laryngoscope 2010;120:2141-6.

30. Woodworth BA, Prince A, Chiu AG, et al. Spontaneous CSF leaks: a paradigm for definitive repair and management of intracranial hypertension. Otolaryngol Head Neck Surg 2008;138:715-20.

31. DeConde AS, Suh JD, Ramakrishnan VR. Treatment of cerebrospinal fluid rhinorrhea. Curr Opin Otolaryngol Head Neck Surg 2015;23:59-64.

32. Zweig JL, Carrau RL, Celin SE, et al. Endoscopic repair of acquired encephaloceles, meningoceles, and meningoencephaloceles: predictors of success. Skull Base 2002;12:133-9.

33. Jennum P, Borgesen SE. Intracranial pressure and obstructive sleep apnea. Chest 
1989;95:279-83.

34. Lund VJ, Stammberger H, Nicolai P, et al. European position paper on endoscopic management of tumours of the nose, paranasal sinuses and skull base. Rhinol Suppl 2010:1-143.

35. Stevens SM, Rizk HG, Golnik K, et al. Idiopathic intracranial hypertension: Contemporary review and implications for the otolaryngologist. Laryngoscope 2017.

36. Hannerz J, Ericson K. The relationship between idiopathic intracranial hypertension and obesity. Headache 2009;49:178-84.

37. Nedelmann M, Kaps M, Mueller-Forell W. Venous obstruction and jugular valve insufficiency in idiopathic intracranial hypertension. J Neurol 2009:256:964-9.

38. Sugerman HJ, Felton WL, 3rd, Salvant JB, Jr., et al. Effects of surgically induced weight loss on idiopathic intracranial hypertension in morbid obesity. Neurology 1995:45:16559.
39. Kupersmith MJ, Gamell L, Turbin R, et al. Effects of weight loss on the course of idiopathic intracranial hypertension in women. Neurology 1998;50:1094-8.

40. Daniels AB, Liu GT, Volpe NJ, et al. Profiles of obesity, weight gain, and quality of life in idiopathic intracranial hypertension (pseudotumor cerebri). Am J Ophthalmol 2007:143:635-41.
Nithin D. Adappa, MD

Division of Rhinology

Department of Otorhinolaryngology-

Head and Neck Surgery

University of Pennsylvania Medical

Center

5th Floor Ravdin Building

3400 Spruce Street

Philadelphia

PA 19104

USA

Tel: +1-215-662-2360

E-mail:

Nithin.Adappa@uphs.upenn.edu 